\title{
A formação de futuros professores de ciência: prática de ensino para desenvolver o pensamento crítico ${ }^{1}$
}

\section{Training prospective science teachers: Teaching practice to develop their critical thinking}

\author{
Ricardo Pereira Sepini²*, Maria Delourdes $\mathrm{Maciel}^{3}$, Ángel Vázquez Alonso ${ }^{4}$
}

\begin{abstract}
RESUMO
Este trabalho apresenta os resultados de uma investigação realizada com graduandos do curso de Ciências Biológicas (Licenciatura) da Universidade Federal de São João del-Rei / Brasil. Como metodologia, ajustou-se a um desenho de pré-teste e pós-teste com os grupos controle e experimental e uma intervenção didática com o grupo experimental. Como instrumentos utilizamos questões do "Cuestionario de opiniones sobre ciencia, tecnología y sociedade" na avaliação pré e pós-teste e na intervenção didática a disciplina prática de ensino de ciências. Os resultados apresentaram diferencias não significativas estatisticamente e algumas possíveis contribuições do pensamento crítico para melhorar a formação de professores são discutidas.
\end{abstract}

Palavras-chave: Aprendizagem; Formação de professores de ciência, Pensamento crítico.

\begin{abstract}
This work presents the results of an investigation carried out with undergraduate students of the Biological Sciences course (Licentiate) at the Federal University of São João del-Rei / Brazil. As a methodology, it was adjusted to a pre-test and post-test design with the control and experimental groups and a didactic intervention with the experimental group. As instruments we used questions from "Questionnaire of opinions on science, technology and society" for pre- and post-test assessment and the didactic intervention in the practical discipline of science teaching. The results showed non-significant differences, and some possible contributions of critical thinking in teacher training to improve training are discussed.
\end{abstract}

Keywords: Learning; Science teachers training; Critical Thinking.

\footnotetext{
${ }^{1}$ Projeto EDU2015-64642-R (MINECO/FEDER) com financiamento do Ministério da Economia e Competitividade da Espanha e do Fundo Europeu de Desenvolvimento Regional.

2 Universidade Federal de São João Del-Rei (UFSJ). *E-mail: ricardopsepini@ufsj.edu.br

${ }^{3}$ Universidade Cruzeiro do Sul (UNICSUL). E-mail: delourdes.maciel@ gmail.com

${ }^{4}$ Universidad de las Islas Baleares (UIB/Espanha). E-mail: angel.vazquez@uib.es
} 


\section{INTRODUÇÃO}

A prática de ensino de Ciências, como toda proposta de formação de docente que visa mudança na prática pedagógica de futuros educadores, deve estar atrelada às preocupações de que os licenciandos tomem consciência de que sua prática envolve, além de observação, reflexão crítica e reorganização de suas ações (SEPINI; MACIEL, 2021).

O papel mais importante a ser cumprido pelo ensino numa perspectiva educacional abrangente é o de habilitar o futuro professor em sua formação inicial a compreender a realidade da Natureza da Ciência e da Tecnologia (NdC\&T) de forma crítica e consciente nos debate e decisões que permeiam a sociedade na qual se encontra inserido. Para este propósito as questões sobre a Ciência e Tecnologia avaliam como eles validam os seus conhecimentos e funcionam no mundo (MACIEL, BISPO-FILHO, GUAZZELLI, 2010).

Para que essa consciência se desenvolva os formadores devem recorrer a estratégias que direcionam os futuros professores(as) para uma ação mais comprometida e crítica com sua formação acadêmica e com o cotidiano das escolas e dos alunos, a fim de que possam enfrentar os desafios apresentados pela sociedade (SEPINI; MACIEL, 2021).

Nessa perspectiva, o enfoque sobre a Natureza da Ciência e da Tecnologia, deve permitir a percepção da interação da Ciência e da Tecnologia com todas as dimensões da Sociedade, considerando as suas relações recíprocas, oferecendo ao educando e ao educador oportunidades para que eles adquiram uma concepção crítica, ampla e humanista sobre a tecnologia e o meio onde está inserido (PINHEIRO; MATOS; BAZZO, 2007). A NdC\&T inclui aspectos relacionados com a Epistemologia e a Sociologia da Ciência e Tecnologia e as relações entre a CTS (MANASSERO-MAS, 2010; MANASSERO-MAS; VÁZQUEZ-ALONSO, 2019).

Para Pinheiro, Matos e Bazzo (2007) a educação em Ciência, Tecnologia e Sociedade (CTS) pode desenvolver essa postura crítica, constituindo-se numa das vias para a formação inicial de professor e de uma consciência de diversidade de interesses no desenvolvimento do sujeito social, ou seja, que tenha condições de apresentar um pensamento crítico a partir de um conhecimento científico.

A área de estudo em $\mathrm{NdC \& T}$ vem a ser um componente propulsor da Alfabetização Científica em todas as áreas, a qual deve estar incorporada ao conteúdo dos 
currículos de formação de professores e currículos escolares de todos os níveis (VÁZQUEZ-ALONSO; MANASSERO-MAS; ACEVEDO-DIAZ, 2006). Há aqui a percepção profunda de um paradigma onde o educando/sujeito deixa seu status de cognoscente, que se relaciona com objetos, e passa a estabelecer novas relações intersubjetivas para a construção de um entendimento e, por conseguinte, de um conhecimento científico que inclui a reação, reelaborando-o, ampliando-o, enfim, criando possibilidades de ação no seu contexto sociocultural (PINHEIRO; MATOS; BAZZO, 2007).

Para enfrentar os atuais desafios das sociedades do conhecimento, tais como o crescente impacto científico e tecnológico, a acelerada inovação digital e informacional, globalização e emergência ecológica, os sistemas educacionais estão sendo solicitados a ensinar as chamadas habilidades do século XXI, que incluem pensamento crítico, comunicação, colaboração, criatividade, empreendedorismo, habilidades de pesquisa, argumentação, análise, interpretação, criatividade, inovação, tomada de decisão e resolução de problemas (VÁZQUEZ-ALONSO; MANASSERO-MAS, 2020).

Esta nova visão de formação docente propõe que os problemas sejam reconhecidos e definidos como informação relevante, contrastando-os com as possíveis alternativas de solução, em que devem ser considerados tanto os resultados positivos quanto os negativos (GÓMEZ; RESTREPO, 2020).

Existem múltiplas definições de pensamento crítico cuja análise permite afirmar que o pensamento crítico é um conjunto de processos que se implementam intencionalmente, com o objetivo de estabelecer conclusões sobre diferentes temas e determinar como, ao aplicar os processos associados ao pensamento crítico, os dados relacionados ou problemas a resolver, são de compostos, sintetizados e avaliados reflexivamente para se chegar a uma conclusão ou solução sobre estes, ou seja, esses processos de pensamento são provisórios e estão em constante revisão, como a própria ciência (ORTEGA-QUEVEDO; PUENTE; RAPP; LÓPEZ-LUENGO, 2020). O pensamento crítico refere-se a processos metacognitivos complexos para pensar bem, o que envolve elementos afetivos, tais como as atitudes (SEPINI; MACIEL, 2021).

Com base nesses dois componentes - cognitivo e afetivo - as decisões são tomadas, os problemas são resolvidos e ocorre interação. Acrescentamos a esses pontos que o pensador crítico deve ter a capacidade de estar ciente de suas próprias afeições ou sentimentos e de como estes, a qualquer momento ou em algum contexto particular, 
podem influenciar sua tomada de decisão (RUIZ; OLIVAR; PRINCE; VÁZQUEZALONSO, 2020). Para Cabral e Maciel (2014), é uma estratégia diferenciada, que pode ser prazerosa e dinâmica, podendo ser realizada com diversos materiais, ferramentas atuais e a exploração de linguagens diferenciadas. Para Manassero e Vázquez (2019), a definição de Pensamento Crítico, envolve: pensamento criativo, claro e preciso em suas justificativas e conclusões, que também avalia e julga meticulosamente todos os seus elementos.

Nesta perspectiva essa pesquisa tem como objetivo investigar possíveis contribuições de uma intervenção didática pautada no pensamento crítico, na disciplina prática de ensino de ciências, a fim de promover ações concretas para a formação de professores(as).

\section{METODOLOGIA}

Esse trabalho se ajusta a um desenho de pré-teste e pós-teste com um grupo controle e um experimental e uma intervenção didática pautada no pensamento crítico na disciplina de Prática de Ensino de Ciências no ano de 2019, realizada com o grupo experimental. Os sujeitos desta pesquisa foram 24 graduandos(as) do $5^{\circ}$ período do curso de Ciências Biológicas (Licenciatura) da Universidade Federal de São João del-Rei do estado de Minas Gerais-Brasil, com faixa etária entre 21 a 27 anos. Destes, 10 compuseram o grupo controle e 14 o grupo experimental.

$\mathrm{Na}$ ementa da disciplina, os pontos essenciais para serem trabalhados são: orientações curriculares oficiais de ciências para o ensino fundamental; metodologias para o ensino de ciências no ensino fundamental; avaliação, produção e utilização de materiais midiáticos para o ensino de ciências; produção e avaliação de modelos didáticos para o ensino de ciências. A disciplina visa orientar o(a) futuro(a) professor(a) na administração do conteúdo a ser desenvolvido em seu trabalho docente, para auxiliá-lo(a) na formação do cidadão.

A metodologia empregada (Quadro 1) nesta investigação foi a aplicação de três questões (pré-teste), como análise prévia, seguido de intervenção pedagógica (disciplina Prática de Ensino de Ciências) e aplicação das questões (pós-teste) para análise final. 
Quadro 1 - Desenho experimental: pré-teste, intervenção pedagógica e pós-teste.

\begin{tabular}{|l|l|l|l|}
\hline \multicolumn{1}{|c|}{ Grupos } & \multicolumn{1}{|c|}{ Pré-teste } & Intervenção Pedagógica & \multicolumn{1}{c|}{ Pós-teste } \\
\hline Grupo Controle & Dia zero & -------- & Dia Final \\
Grupo Experimental & Dia zero & 4 meses & Dia Final \\
\hline
\end{tabular}

Fonte: Os autores.

A intervenção pedagógica foi realizada na disciplina Prática de Ensino de Ciências, de natureza obrigatória, que possui um total de 36 horas/aulas, cujo objetivo é instrumentalizar os(as) licenciandos (as) para a prática docente no Ensino Fundamental. As atividades de intervenção com o grupo experimental envolveram temas (Quadro 2) associados à sua formação acadêmica, além de temas como, resolução de problemas e os processos envolvidos nas conclusões ou solução dos mesmos, considerando-se que tais processos de pensamento são provisórios e estão em constante revisão, como a própria ciência.

Quadro 2 - Intervenção pedagógica e data.

\begin{tabular}{|c|c|}
\hline Estrutura & Temáticas \\
\hline Dia Zero & $\begin{array}{l}\text { Aplicação COCTS (Grupo Controle e Grupo } \\
\text { Experimental) }\end{array}$ \\
\hline $\begin{array}{l}\text { Intervenção Pedagógica } \\
\text { (Grupo Experimental) }\end{array}$ & $\begin{array}{l}\text { Por que ensinar Ciências no Ensino Fundamental } \\
\text { (Orientação Curricular) } \\
\text { Necessidades formativas do professor de Ciências } \\
\text { Linguagem e a Construção do conhecimento em } \\
\text { Ciências } \\
\text { Alfabetização Científica } \\
\text { Seleção de conteúdos no Ensino Fundamental } \\
\text { (Conteúdos Integradores) } \\
\text { Avaliação do Ensino } \\
\text { Modalidades didáticas e recursos } \\
\text { Experimentação e Ensino de Ciências } \\
\text { Mídia }\end{array}$ \\
\hline
\end{tabular}




\begin{tabular}{|l|l|} 
& $\begin{array}{l}\text { Leituras e Ensino de Ciências } \\
\text { Jogos, modelos, simulações } \\
\text { Projetos no Ensino de Ciências } \\
\text { Resolução de problemas no Ensino Fundamental } \\
\text { Ciência, Tecnologia e Sociedade no Ensino Fundamental }\end{array}$ \\
\hline Dia Final & $\begin{array}{l}\text { Aplicação COCTS (Grupo Controle e Grupo } \\
\text { Experimental) }\end{array}$ \\
\hline
\end{tabular}

Fonte: Os autores.

Como aporte teórico para a intervenção pedagógica, utilizamos os referenciais apresentado no Quadro 3.

Quadro 3 - Referenciais utilizados na intervenção pedagógica.

\begin{tabular}{|c|c|}
\hline Autor & Obra \\
\hline $\begin{array}{l}\text { BRASIL } \\
(1997)\end{array}$ & Parâmetros Curriculares Nacionais: Ciências Naturais \\
\hline $\begin{array}{l}\text { BRASIL } \\
(2008)\end{array}$ & Ciências da Natureza, Matemática e suas Tecnologia \\
\hline $\begin{array}{l}\text { MINAS GERAIS } \\
(2007)\end{array}$ & Conteúdo Básico Comum \\
\hline $\begin{array}{l}\text { APPLE } \\
\text { (1992) }\end{array}$ & Ideologia e Currículo \\
\hline $\begin{array}{l}\text { BACHELARD } \\
(2008)\end{array}$ & $\begin{array}{l}\text { A formação do Espírito Científico: Contribuição para uma } \\
\text { Psicanálise do Conhecimento }\end{array}$ \\
\hline $\begin{array}{l}\text { CHASSOT } \\
\text { (1998) }\end{array}$ & Ciência, Ética e Cultura na Educação \\
\hline $\begin{array}{l}\text { KRASILCHIK } \\
(2004)\end{array}$ & Prática de Ensino de Biologia \\
\hline $\begin{array}{l}\text { SANTOS } \\
(1998)\end{array}$ & Um discurso sobre as Ciências \\
\hline $\begin{array}{l}\text { BAZZO; } \\
\text { LINSINGEN; } \\
\text { PEREIRA } \\
(2003)\end{array}$ & Introdução aos Estudos CTS \\
\hline
\end{tabular}


Fonte: Os autores.

Com o grupo controle não houve intervenção. Para análise do pensamento crítico utilizamos como instrumento avaliativo três questões do COCTS (Cuestionario de Opiniones sobre Ciencia, Tecnología y Sociedad) em sua versão traduzida para a português do Brasil. Os alunos devem aplicar habilidades de pensamento crítico para decidir o seu grau de concordância com cada uma das frases incluídas nas questões com base no conteúdo da NdC\&T desenvolvidos na intervenção didática.

O COCTS é um instrumento amplo em conteúdo, flexível em sua aplicação, válido e confiável estatisticamente para a investigação. As três questões do COCTS utilizadas são aquelas que apresentam uma relação com a ciência, a tecnologia e com a sociedade (CTS).

Utilizamos as questões 10111, 10113 e 10211. Na tabela 1 apresentamos a estrutura da questão 10111. Para análise estatística das questões utilizamos o teste estatístico Wilcoxon.

Tabela 1. Questão 10111.

\begin{tabular}{|c|c|}
\hline 10111 & $\begin{array}{l}\text { Definir o que é ciência é difícil porque a ciência é complexa e engloba muitas coisas. } \\
\text { mas a ciência é principalmente: }\end{array}$ \\
\hline A. & o estudo de campos como biologia, química, geologia e física. \\
\hline B. & $\begin{array}{l}\text { um corpo de conhecimento, como princípios, leis e teorias que explicam o mundo ao } \\
\text { nosso redor (matéria, energia e vida). }\end{array}$ \\
\hline C. & $\begin{array}{l}\text { explorar o desconhecido e descobrir coisas novas sobre o mundo e o universo e como } \\
\text { elas funcionam. }\end{array}$ \\
\hline D. & $\begin{array}{l}\text { realizar experimentos para resolver problemas de interesse sobre o mundo que nos } \\
\text { rodeia. }\end{array}$ \\
\hline E. & $\begin{array}{l}\text { inventar ou projetar coisas (por exemplo, corações artificiais, computadores, veículos } \\
\text { espaciais). }\end{array}$ \\
\hline F. & $\begin{array}{l}\text { buscar e usar o conhecimento para tornar este mundo um lugar melhor para se viver (por } \\
\text { exemplo, curar doenças, consertar a poluição e melhorar a agricultura). }\end{array}$ \\
\hline G. & $\begin{array}{l}\text { uma organização de pessoas (chamadas cientistas) que têm ideias e técnicas para } \\
\text { descobrir novos conhecimentos. }\end{array}$ \\
\hline H. & um processo investigativo sistemático e o conhecimento resultante. \\
\hline I. & você não pode definir ciência. \\
\hline
\end{tabular}

Fonte: os autores.

ANÁLISE E DISCUSSÃO DOS RESULTADOS 
Todo o processo de intervenção pedagógica realizada durante a disciplina Prática de Ensino de Ciências, foram voltadas há promover ações concretas para a formação de professores, pautada na aprendizagem do pensamento crítico.

Durante o processo, um fator que apareceu com muita expressão é a aprendizagem. Sobre a aprendizagem Freire (2009) descreve que ela deve estar de maneira integrada ao processo formativo, pois "ensinar não é transferir conhecimento, mas criar as possibilidades para a sua própria produção ou a sua construção”.

Conforme apresentado na tabela 2 as respostas dos(as) graduandos(as) do grupo controle não apresentaram diferenças estatisticamente significativas. Nesta perspectiva, fica evidente que os futuros professores não conseguiram assimilar, aprender a interação com a prática de ensino, visando um pensamento crítico, e ao mesmo tempo é importante salientar que os futuros professores deste grupo não participaram da intervenção pedagógica.

Tabela 2. Resultado estatístico das questões do cocts.

\begin{tabular}{|c|c|c|c|c|c|c|c|c|}
\hline \multirow{2}{*}{ Questão } & \multicolumn{3}{|c|}{ Grupo Controle N=10 } & \multicolumn{3}{c|}{ Grupo Experimental N=14 } \\
\cline { 2 - 8 } & $\begin{array}{c}\text { Pré- } \\
\text { teste/Média }\end{array}$ & $\begin{array}{c}\text { Pós- } \\
\text { teste/Média }\end{array}$ & $\mathbf{T}^{\mathbf{5}}$ & $\mathbf{p}^{\mathbf{6}}$ & $\begin{array}{c}\text { Pré- } \\
\text { teste/Média }\end{array}$ & $\begin{array}{c}\text { Pós- } \\
\text { teste/Média }\end{array}$ & $\mathbf{T}$ & $\mathbf{p}$ \\
\hline 10111 & 0.002 & 0.034 & 10 & 0.507 & 0.176 & 0.144 & 1 & $0.028^{7}$ \\
10113 & -0.020 & -0.204 & 12 & 0.114 & 0.119 & 0.113 & 7 & 0.463 \\
10211 & -0.001 & -0.064 & 24 & 0.721 & 0.061 & 0.028 & 4 & 0.091 \\
\hline
\end{tabular}

Fonte: Os autores.

Conforme apresentado na tabela 2 as respostas dos(as) graduandos(as) do grupo controle não apresentaram diferenças estatisticamente significativas. Nesta perspectiva, fica evidente que os futuros professores não conseguiram assimilar, aprender a interação com a prática de ensino, visando um pensamento crítico, e ao mesmo tempo é importante salientar que os futuros professores deste grupo não participaram da intervenção pedagógica.

\footnotetext{
5 T: valor calculado da estatística.

${ }^{6}$ p: probabilidade exata calculada.

${ }^{7}$ Apresenta diferença estatisticamente significativa.
} 
No grupo experimental (tabela 2) somente a questão 10111 apresentou diferença estatisticamente significativa $\mathrm{p}<0.05$. Esse resultado para a questão 10111, vai de encontro com os autores Ruiz, Olivar, Prince e Vázquez-Alonso (2020), onde acrescenta a esse ponto que o pensador crítico deve ter a capacidade de estar ciente de suas próprias afeições ou sentimentos e de como estes, a qualquer momento ou em algum contexto particular, podem influenciar sua tomada de decisão.

Atividades desta magnitude realizada na prática de ensino de ciências com enfoque no pensamento crítico, podem nos apresentar o começo de uma direção para uma compreensão de como os(as) graduandos(as), após serem indagados sobre questões referentes a esse processo, analisam as aplicações, discutindo problemas e possíveis soluções na sociedade (SEPINI; MACIEL, 2021).

\section{CONCLUSÃO}

Os resultados obtidos não mostram uma melhoria no conhecimento de NdC\&T no grupo experimental como seria de se esperar após o tratamento, que podem estar relacionados com a não apropriação de um processo educativo em que aprender pensamento crítico, seja uma forma de ampliar as condições para o exercício da cidadania e/ou que o instrumento de análise (COCTS), seja impreterivelmente utilizado para análise do conhecimento da Natureza da Ciências e da Tecnologia e não para o pensamento crítico. Um outro ponto a ser investigado, é a intervenção pedagógica, onde a temática, possa estar desassociada de uma visão voltada para se trabalhar o pensamento crítico em futuros professores de ciências.

Ao mesmo tempo, acreditamos que atividades desta magnitude, que visam apresentar uma temática de forma problematizadora e relacionada com o contexto onde esses graduandos irão atuar na prática de ensino, com um período de curto e médio prazo de realização, trazem concepções positivas da temática de pensamento crítico para esses(as) licenciandos em sua formação.

A partir desta análise pode ser possível realizar mudanças de habilidades de pensamento crítico nos graduandos, mas para que essas mudanças sejam mais positivas, necessita-se de uma melhor apropriação da estratégia utilizada. Para tanto, se faz necessária a realização de novas pesquisas para o aperfeiçoamento e ampliação dos instrumentos utilizados na intervenção e na avaliação. 
Diante disso, formar professores críticos, capacitados e habilitados para desenvolver aspectos relacionados ao pensamento crítico continua a ser uma meta importante para uma educação de qualidade em que os estudantes entendam e compreendam o exercício para a cidadania e apliquem em seu cotidiano de maneira refletida e consciente.

\section{REFERÊNCIAS}

APPLE, M. W. Ideologia e Currículo. São Paulo: Brasiliense, 1982.

AYRES, M.; JUNIOR, M. A.; AYRES, L. D.; SANTOS, A. A. S. BioEstat5.3: aplicações nas áreas das ciências Bio-Médicas. Belém: UFPA, 2007.

BACHELARD, G. A formação do espírito científico: contribuição para uma psicanálise do conhecimento. Rio de Janeiro: Contraponto, 2008.

BAZZO, W. A.; LINSINGEN, I. V.; PEREIRA, L. T. V. Introdução aos estudos CTS (Ciência, Tecnologia e Sociedade. Madri, Espanha: OEI (Organização dos Estados Ibero-americanos), 2003.

BRASIL. Secretaria de Educação Fundamental. Parâmetros curriculares nacionais: ciências naturais. Brasília, 1997.

. Secretaria de Educação Básica. Ciências da natureza, matemática e suas tecnologia. Brasília: Ministério da Educação, 2008.

CABRAL, S. A., MACIEL, M. D. Natureza da ciência e da tecnologia em sistemas de classificação biológica: experiência de ensino e aprendizagem com emprego de uma sequência didática com jogos pedagógicos. 2014. 256 f. Dissertação (Mestrado em Ensino de Ciência) - Universidade Cruzeiro do Sul, São Paulo, 2014.

CHASSOT, A.; OLIVEIRA, R.J. Ciência, ética e cultura na educação. São Leopoldo: UNISINOS, 1998.

FREIRE, P. Pedagogia da autonomia: saberes necessários à prática educativa. 39. ed. São Paulo: Paz e Terra, 2009.

GÓMEZ, Y. P. V., RESTREPO, M. M. C. Compuesto inorgánicos en el ambiente. Secuencia de enseñanza y aprendizaje (SEA) para desarrollar pensamiento crítico en su aprendizaje. Tecné Episteme y Didáxis, n. 48, p. 181-202, 2020.

KRASILCHIK, M. Prática de Ensino de Biologia. São Paulo: Edusp, 2004.

MACIEL, D. M; BISPO-FILHO, D. O.; GUAZZELLI, I. R. B. Relatório de Pesquisa. Proyecto Iberoamericano de Evaluación de Actitudes Relacionadas con la Ciencia, la Tecnología y la Sociedad (PIEARCTS), Brasil, 2010. 
MANASSERO-MAS, M. A. El proyecto Iberoamericano de evaluación de actitudes relacionadas con la ciencia, la tecnología y la sociedad (PIEARCTS): un estudio de investigación cooperativa. In: MACIEL, D. M.; AMARAL, C. L. C.; GUAZZELLI, I. R. B. (Eds.), Ciência, Tecnologia \& Sociedade: pesquisa e ensino. São Paulo: Terracota, 2010, p. 13-42.

MANASSERO, M.A.; VÁZQUEZ, A. Taxonomía de las destrezas de pensamiento: una herramienta clave para la alfabetización científica. In: MACIEL, D. M.; ALBRECHT, E. (org.), Ciência, Tecnologia y Sociedade: Ensino, Pesquisa e Formação. São Paulo: UNICSUL, 2019, p. 17-38.

MANASSERO-MAS, M.A.; VÁZQUEZ-ALONSO, A. Conceptualización y taxonomía para estructurar los conocimientos acerca de la ciencia. Revista Eureka sobre Enseñanza y Divulgación de las Ciencias, v. 16, n. 3, p. 3104-3117, 2019.

MELO, T. B.; CHRISPINO, A. Ideologia da certeza Matemática: Contribuições Reflexivas do Enfoque CTS. Revista Ciências \& Ideias. v. 5, n. 2, p.100-119, 2014.

MINAS GERAIS. Secretaria do Estado de Educação. Conteúdo Básico Comum: CBC Física. Belo Horizonte: SEE, 2007.

ORTEGA-QUEVEDO, V.; PUENTE, C. G.; RAPP, C. V.; LÓPEZ-LUENGO, M. A. Diseño y validación de instrumentos de evaluación de pensamiento crítico en educación primaria. Tecné Episteme y Didáxis, n. 48, p. 91-110, 2020.

PINHEIRO, N. A. M.; MATOS, E. A. S. A.; BAZZO, W. A. Refletindo acerca da ciência, tecnologia e sociedade: enfocando o ensino médio. Revista Ibero Americana de Educação, n. 44, p. 147-165, 2007.

RUIZ, M. G.; OLIVAR, V. G.; SOCORRO, J. G. R.; VÁQUEZ-ALONSO, Á. Los efectos de la temática socioambiental en las habilidades de pensamiento crítico del futuro profesorado de primaria. Tecné Episteme y Didáxis, n. 48, p. 75-90, 2020.

SANTOS, B. S. Um discurso sobre as ciências. São Paulo: Cortez, 1998.

SEPINI, R. P.; MACIEL, M. D. Prática de ensino como estratégia para proporcionar o pensamento crítico em futuros professores de ciências. In: Congreso Internacional sobre Investigación em la Didáctica de las Ciências, XI., 2021, Lisboa. Anais... Lisboa: Revista Enseñanza de las Ciencias, 2021. p. 1829-1832.

VÁSQUEZ-ALONSO. Á.; MANASSERO-MAS, M. A. Las destrezas de pensamiento y las calificaciones escolares en educación secundaria: validación de un instrumento de evaluación libre de cultura. Tecné Episteme y Didáxis, n. 48, p. 33-54, 2020.

VÁZQUEZ-ALONSO, A.; MANASSERO-MAS, M. A. Una secuencia de enseñanzaaprendizaje sobre un tema socio-científico: análisis y evaluación de su aplicación en el aula. Revista Didáctica de la Química, v. 25, n.1, p. 190-202, 2014. 
VÁZQUEZ-ALONSO, A.; MANASSERO-MAS, M. A.; ACEVEDO-DIAZ, J. A. An Analysis of Complex Multiple-Choice Science-Technology-Society Items:

Methodological Development and Preliminary Results. Science Education, v. 90, n. 4, p. 681-706, 2006.

Recebido em: 01/10/2021

Aprovado em: 30/10/2021

Publicado em: 10/11/2021 